\title{
An Invariant of Colored Links via Skein Relation
}

\author{
Francesca Aicardi ${ }^{1}$
}

To the memory of Serezha Duzhin

Received: 30 May 2015 / Accepted: 14 December 2015 / Published online: 1 March 2016

(C) Institute for Mathematical Sciences (IMS), Stony Brook University, NY 2016

\begin{abstract}
In this note, we define a polynomial invariant for colored links by a skein relation. It specializes to the Jones polynomial for classical links.
\end{abstract}

Keywords Knot theory $\cdot$ Knots invariants $\cdot$ Colored links

Mathematics Subject Classification 57M25

\section{Introduction}

This note deals with an invariant of colored links. Let us detail these objects.

An oriented link with $n$ components is a set of $n$ disjoint smooth oriented closed curves embedded in $S^{3}$. An oriented link is colored if each component is provided with a color. In other words, a link is colored if a function $\gamma$ is defined on the set of components with values in a finite set $N$ of colors. Let's call such a function $\gamma$ a coloration. Every coloration introduces an equivalence relation in the set of components: two components are equivalent if they have the same color. Let $C$ be the set of components of a link $L$. Two colorations $\gamma$ and $\gamma^{\prime}$ of $L$ are said to be equivalent if there is a bijection in $N$ between the images $\gamma(C)$ and $\gamma^{\prime}(C)$, which takes, for every $c \in C$, the color $\gamma(c)$ into the color $\gamma^{\prime}(c)$. Two colorations define the same partition of the set $C$ into classes of equivalence if and only if they are equivalent.

An invariant of colored links takes the same value on isotopic links with the same coloration, and may take different values on the same link with different colorations.

Francesca Aicardi

faicardi@ictp.it

1 The Abdus Salam International Centre for Theoretical Physics (ICTP), Strada Costiera, 11, 34151 Trieste, Italy 
Our invariant of colored links takes the same value on isotopic links with equivalent colorations.

There is a wide literature on the invariants of colored links developed from the multivariable Alexander polynomial, which includes contributions by Fox, Conway, Kauffman, and many others. For a summary of the history of these invariants see Cimasoni (2004). More recent works in this direction (Murakami 1992; Cimasoni 2004; Cimasoni and Florens 2008) use also skein relations for the Alexander polynomial, but the colors of the components of the links involved in such skein relations are unaltered.

The skein relation introduced in the present note may change the colors of the components of the link. Therefore, the invariant that we define seems to be new; still, the relation of it with the known invariants has to be investigated.

We recall here the definition of an invariant of classical links by a skein relation.

Among the most famous topological invariants of oriented knots and links, we single out the Alexander polynomial, introduced in Alexander (1928), and the Jones polynomial, introduced in Jones (1987). Both these polynomials in one variable can be considered as particular instances of a more general invariant of isotopy classes of oriented links, the so-called HOMFLY polynomial, introduced in Freyd et al. (1985), which is a Laurent polynomial in two variables. This polynomial is uniquely defined by the condition that its value is 1 on the unknotted oriented circle and by a skein relation, as we explain below.

Suppose that $K_{\mathrm{x}}^{+}, K_{\mathrm{x}}^{-}$and $K_{\mathrm{x}}^{0}$ are diagrams of three oriented links that are exactly the same except in the neighborhood of a crossing $\mathrm{X}$, where they look as shown in the figure below. Then the skein relation is an equation that relates the values of the polynomial $P$ on these links:

$$
\ell P\left(K_{\mathrm{x}}^{+}\right)+\ell^{-1} P\left(K_{\mathrm{x}}^{-}\right)+m P\left(K_{\mathrm{x}}^{0}\right)=0
$$
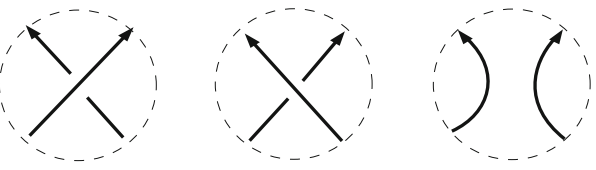

The proof that the skein relation allows to define the value of $P$ on any link is done by induction on the number of crossings. By an ordered sequence of crossing changes, any link $K$ can be changed into a trivial link $K_{u}$, i.e. a collection of unknotted and unlinked components. The value of $P$ on $K_{u}$ is supposed known: indeed, it must be independent of the number $n$ of crossings, depending only on the number of components. Let $\left(\mathbf{x}_{1}, \ldots, \mathbf{x}_{m}\right)$ be the sequence of crossings, where the sign has to be changed to transform $K$ into $K_{u}$, and let ${ }_{i} K$ be the diagram obtained from $K$ by changing the sign of the first $i$ crossings, so that ${ }_{m} K=K_{u}$. The skein relation is thus applied to the crossing $\mathrm{x}_{1}$ : if this crossing is positive in $K$, then $K=K_{\mathrm{x}_{1}}^{+}$, and one obtains $P\left(K_{\mathrm{x}_{1}}^{+}\right)$as a linear combination of the values $P\left(K_{\mathrm{x}_{1}}^{-}\right)$and $P\left(K_{\mathrm{x}_{1}}^{0}\right)$. Since $K_{\mathrm{x}_{1}}^{0}$ has $n-1$ crossings, $P\left(K_{\mathrm{x}_{1}}^{0}\right)$ is known by the induction hypothesis. Now, by definition, $K_{\mathrm{x}_{1}}^{-}={ }_{1} K$, and the 
value $P\left({ }_{1} K\right)$ is calculated by using the skein relation applied to the second crossing $\mathbf{X}_{2}$, and so on, until the last crossing change, in which either ${ }_{(m-1)} K_{\mathbf{x}_{m}}^{+}$or $_{(m-1)} K_{\mathbf{X}_{m}}^{-}$ coincides with $K_{u}$. Then the calculation of $P(K)$ is concluded, being obtained from $P\left(K_{u}\right)$ and the values of $P$ on $(m-1)$ links with $n-1$ crossings.

It is proved that $\mathfrak{P}(K)$ calculated in this way is independent of the chosen sequence of changes, as well as independent of the particular projection of the link. The polynomial $P$ is in fact an isotopy invariant of the link.

Suppose now that we want to define by a skein relation an invariant, which is able to distinguish two links that are identical, but have non-equivalent colorations. We show that it is possible to obtain the invariant we want by introducing a new skein relation, which takes into account strands of any color, and by setting the value of the invariant on the trivial links with non-equivalent colorations, i.e., on collections of $n$ unknotted and unlinked components that are colored, for every $c=1, \ldots, n$, with $c$ different colors.

Our invariant, $F$, requires three variables, say $x, w$, and $t$. When the link is colored with a sole color, our invariant is reduced to another instance of the HOMFLY polynomial and becomes the Jones polynomial in the variable $t$ if $w=t^{1 / 2}$.

Finally, we remark that the polynomial $F$ was not found by searching for an invariant for colored links. Its definition is a byproduct of the study of another class of links, the tied links (see Aicardi and Juyumaya 2015), obtained from the closure of tied braids, which, in turn, constitute a diagrammatic representation of an abstract algebra, the socalled algebra of braids and ties. The defining set of generators of the algebra of braids and ties consists of two sets of generators, one of them is interpreted as the set of usual braid generators and the other one as the set of ties. Despite the fact that the defining relations, which involve tie generators, look complicated and, in particular, ties do not commute with braids, we have shown (Aicardi and Juyumaya 2014) that a new geometric interpretation of the tie generators, coherent with the defining relations of the algebra, allows in particular to reduce ties to simple connections between different strands of braids, and, under closure, between different components of links. The set of connections behaves as an equivalence relation among the link components. As a consequence, the invariant polynomial for tied links defined in Aicardi and Juyumaya (2015) provides an invariant of colored links as well.

\section{An Invariant for Colored Links}

A colored link diagram looks like a diagram of a link, where each component is colored.

Definition 1 Two oriented colored links are c-isotopic if they are ambient isotopic and their colorations are equivalent (Fig. 1).

Let $R$ be a commutative ring, and $\mathcal{C}$ be the set of colored oriented link diagrams. By an invariant of colored links, we mean a function $I: \mathcal{C} \rightarrow R$, which is constant on each class of c-isotopic links.

Remark 1 In the sequel, we denote by $\mathrm{K}$ an oriented colored link as well as its diagram, if there is no risk of confusion. 

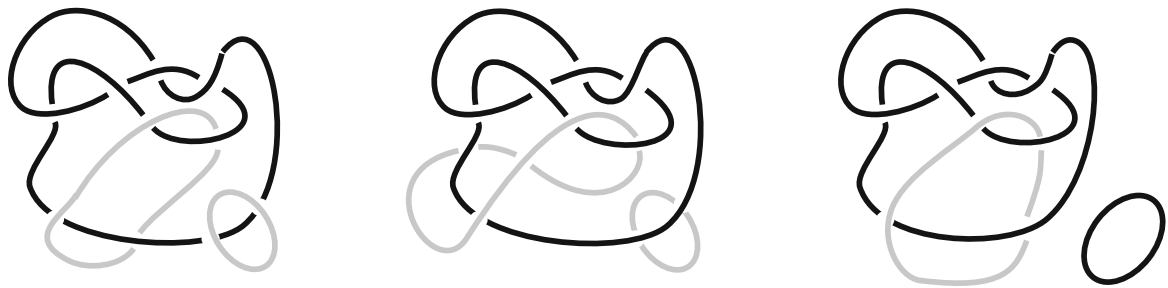

Fig. 1 The first two diagrams represent c-isotopic colored links, the third one is not c-isotopic to the others. Black and gray represent two different colors

a

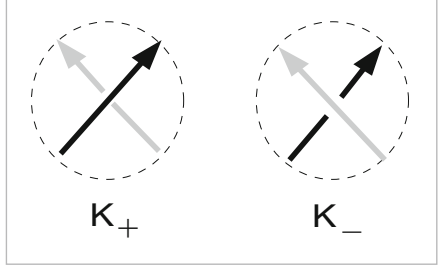

b

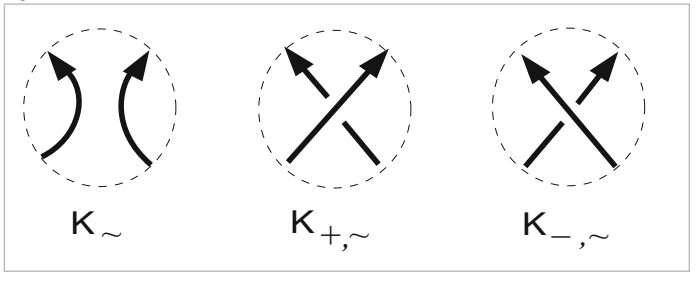

Fig. 2 a The discs, where $\mathrm{K}_{+}$and $\mathrm{K}_{-}$are not c-isotopic. $\mathbf{b}$ The discs, where $\mathrm{K}_{\sim}, \mathrm{K}_{+}, \sim$, and $\mathrm{K}_{-, \sim \text { are not }}$ c-isotopic. Black and gray indicate any two colors

Theorem 1 There exists a rational function in the variables $x, w, t, F: \mathcal{C} \rightarrow$ $\mathbb{Q}(x, t, w)$, invariant of oriented colored links, uniquely defined by the following three conditions on colored-link diagrams:

I The value of $F$ is equal to 1 on the unknotted circle.

II Let $\mathrm{K}$ be a colored link with $n$ components and c colors. By [K; O], we denote the colored link with $n+1$ components consisting of $\mathrm{K}$ and the unknotted and unlinked circle colored with the $(c+1)$-st color. Then

$$
F([\mathrm{~K} ; \mathrm{O}])=\frac{1}{w x} F(\mathrm{~K})
$$

III (skein relation) Let $\mathrm{K}_{+}$and $\mathrm{K}_{-}$be the diagrams of two colored links that are the same outside a small disc into which two strands enter, and inside this disc look as shown in Fig. 2a. Black and gray indicate any two colors, not necessarily distinct. Similarly, let $\mathrm{K}_{\sim}$ and $\mathrm{K}_{+, \sim}$ be two links that inside the disc look as shown in Fig. $2 \mathrm{~b}$, while outside the disc coincide with $\mathrm{K}_{+}$and $\mathrm{K}_{-}$, except, possibly, for the colors of all the components of the link having the same colors as the strands entering the disc; all these components in $\mathrm{K}_{\sim}$ and $\mathrm{K}_{+, \sim}$ are colored by a sole color. Then the following identity holds:

$$
\frac{1}{w} F\left(\mathrm{~K}_{+}\right)-w F\left(\mathrm{~K}_{-}\right)=\left(1-t^{-1}\right) F\left(\mathrm{~K}_{\sim}\right)+\frac{1}{w}\left(1-t^{-1}\right) F\left(\mathrm{~K}_{+, \sim}\right) .
$$


Remark 2 Skein relation III holds for any two colors of the strands involved. In par-

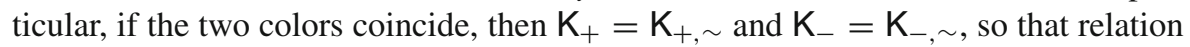
III is reduced to relation

IV

$$
\frac{1}{t w} F\left(\mathrm{~K}_{+, \sim}\right)-w F\left(\mathrm{~K}_{-, \sim}\right)=\left(1-t^{-1}\right) F\left(\mathrm{~K}_{\sim}\right)
$$

Relation III also implies the following two relations; they will be used in the sequel. $\mathrm{Va}$

$$
\frac{1}{w} F\left(\mathrm{~K}_{+}\right)=w\left[F\left(\mathrm{~K}_{-}\right)+(t-1) F\left(\mathrm{~K}_{-, \sim}\right)\right]+(t-1) F\left(\mathrm{~K}_{\sim}\right) .
$$

$\mathrm{Vb}$

$$
w F\left(\mathrm{~K}_{-}\right)=\frac{1}{w}\left[F\left(\mathrm{~K}_{+}\right)+\left(t^{-1}-1\right) F\left(\mathrm{~K}_{+, \sim}\right)\right]+\left(t^{-1}-1\right) F\left(\mathrm{~K}_{\sim}\right) .
$$

Remark 3 As an example of using relations I, II, and III, let's calculate the value of $F$ on the unlink $\mathrm{O}_{n}^{c}$ consisting of $n$ circles and $c$ colors. If $n=c$, then, by II,

$$
F\left(\mathrm{O}_{c}^{c}\right)=\frac{1}{w x} F\left(\mathrm{O}_{c-1}^{c-1}\right)=\left(\frac{1}{w x}\right)^{c-1}
$$

If $n>c$, then there are two circles colored by the same color. Regard them as $\mathrm{K}$. Then $\mathrm{K}_{+}=\mathrm{K}_{-}=\mathrm{O}_{n-1}^{c}$ (see figure).
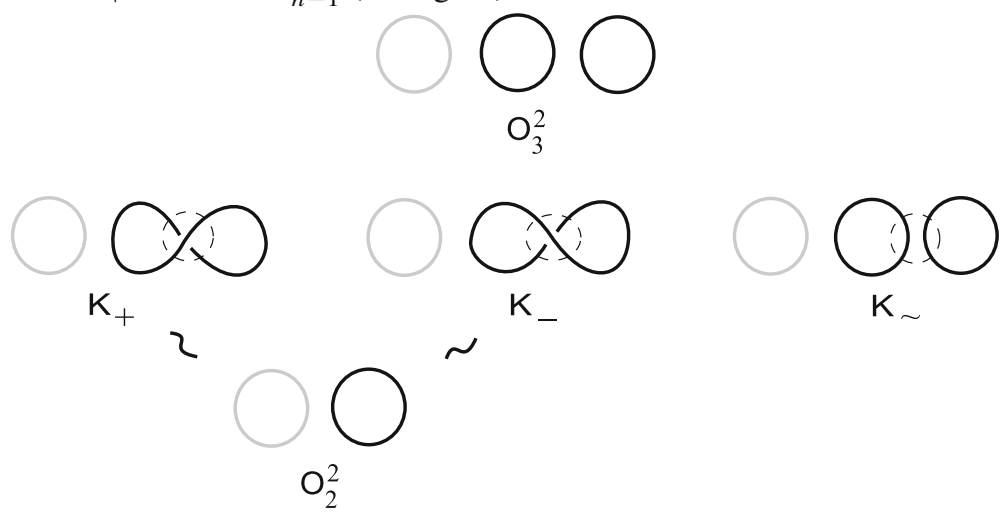

By relation IV, $\left(1-t^{-1}\right) F\left(\mathrm{O}_{n}^{c}\right)=\left(\frac{1}{t w}-w\right) F\left(\mathrm{O}_{n-1}^{c}\right)$. So,

$$
F\left(\mathrm{O}_{n}^{c}\right)=\frac{1-t w^{2}}{t w\left(1-t^{-1}\right)} F\left(\mathrm{O}_{n-1}^{c}\right)=\frac{t w^{2}-1}{(1-t) w} F\left(\mathrm{O}_{n-1}^{c}\right)=\frac{y}{x w} F\left(\mathrm{O}_{n-1}^{c}\right)
$$


where

$$
y=\frac{x\left(t w^{2}-1\right)}{1-t} .
$$

By induction, $F\left(\mathrm{O}_{n}^{c}\right)=\left(\frac{y}{x w}\right)^{n-c} F\left(\mathrm{O}_{c}^{c}\right)$. Thus,

$$
F\left(\mathrm{O}_{n}^{c}\right)=\frac{y^{n-c}}{(w x)^{n-1}} .
$$

Proof of Theorem 1 Theorem 1 is proved by the procedure used in the proof of the corresponding theorem for classical links stated on page 112 in Lickorish and Millet (1987). We will outline the parts, where the presence of colors modifies the demonstration.

Of course, the Reidemeister moves for colored links are all the Reidemeister moves, where the strands involved may have different colors.

One starts with zero crossings as the induction base: the value of $F$ on the unlink $O_{n}^{c}$ with $n$ circles and $c$ colors is given by $\frac{y^{n-c}}{(w x)^{n-1}}$ in accordance with Remark 3 .

Let $\mathcal{C}^{p}$ be the set of diagrams of oriented colored links with $p$ crossings, and $\mathrm{K} \in \mathcal{C}^{p}$. By ordering the components and fixing a point in each component, one constructs, for every diagram $\mathrm{K}$, an associated standard ascending diagram $\mathrm{K}^{\prime}$ in the following way: traverse the components of $\mathrm{K}$ in their given order and from their base points in the direction specified by their orientation. Every crossing, encountered the first time, is either over or under crossing. In the first case, the crossing is changed; otherwise, it is left as in $\mathrm{K}$. $\mathrm{K}^{\prime}$ consists of the same number of components as $\mathrm{K}$, completely unknotted and unlinked. $\mathrm{K}$ and $\mathrm{K}^{\prime}$ are identical, except for a finite sequence of crossings, which we call 'deciding', where the signs are opposite. Furthermore, we define $\tilde{K}^{\prime}$ as obtained from $\mathrm{K}^{\prime}$ by coloring with a sole color all the components that have the same two colors as the two strands involved into each deciding crossing. $\mathrm{K}^{\prime}$ and $\tilde{\mathrm{K}}^{\prime}$ are, by construction, collections of unknotted and unlinked components; $\mathrm{K}^{\prime}$ has the same number of colors as $\mathrm{K}, \tilde{\mathrm{K}}^{\prime}$ may have a smaller number of colors. The procedure defining $\mathrm{K}^{\prime}$ allows to get an ordered sequence of deciding crossings, whose order depends on the ordering of the components and on the choice of the base points.

The induction hypothesis states that, on $\mathcal{C}^{p}$, we have a well defined function $F$, satisfying relations I-III, which is independent of the ordering of the components, independent of the choices of the base points, and invariant under Reidemeister moves that do not increase the number of crossings beyond $p$. Moreover, the induction hypothesis states that the value of $F$ on any colored link with $p$ crossings, consisting of $n$ components unknotted, unlinked, and colored with $c$ colors $(c \leq n)$, is the same as $F\left(\mathrm{O}_{n}^{c}\right)$ given by $(3)$.

Now, let $\mathrm{K}$ be in $\mathcal{C}^{p+1}$. If $\mathrm{K}$ consists of $n$ unknotted and unlinked components colored with $c(c \leq n)$ colors, let's define

$$
F(\mathrm{~K})=\frac{y^{n-c}}{(w x)^{n-1}}
$$


Otherwise, construct the associate ascending diagrams $\mathrm{K}^{\prime}$ and $\tilde{\mathrm{K}}^{\prime}$ and consider the first deciding crossing $\mathbf{X}$. If, in a neighborhood of $\mathbf{X}$, the colored link looks like $\mathrm{K}_{+, \sim}$ (or $\mathrm{K}_{-, \sim}$ ), use skein relation IV to write the value of $F$ in terms of $\mathrm{K}_{-, \sim}$ and $\mathrm{K}_{\sim}$ (respectively, $\mathrm{K}_{+, \sim}$ and $\mathrm{K}_{\sim}$ ). If, in a neighborhood of $\mathbf{x}$, the colored link looks like $\mathrm{K}_{+}$(respectively, $\mathrm{K}_{-}$), use skein relation $\mathrm{Va}$ (respectively, $\mathrm{Vb}$ ) to write the value of $F$ in terms of the value of $F$ on the colored links $\mathrm{K}_{-}, \mathrm{K}_{-, \sim}$, and $\mathrm{K}_{\sim}$ (respectively, $\mathrm{K}_{+}, \mathrm{K}_{+, \sim}$, and $\left.\mathrm{K}_{\sim}\right)$. Observe that if the diagram $\mathrm{K}_{\epsilon}$ or $\mathrm{K}_{\epsilon \sim \sim}(\epsilon= \pm)$ coincides, in a neighborhood of the crossing $\mathbf{X}$, with the original diagram, then $\mathbf{K}_{-\epsilon}$ or $\mathbf{K}_{-\epsilon, \sim}$ coincides, in the neighborhood of the same crossing, with the associated diagram $\mathrm{K}^{\prime}$ or $\tilde{\mathrm{K}}^{\prime}$ respectively. On the other hand, $\mathrm{K}_{\sim}$ represents a colored link diagram with $p$ crossings, for which the value of $F$ is known and is invariant according to the induction hypothesis. Then we look for the second deciding crossing, which is present in all the diagrams with $p+1$ crossings, obtained by applying the skein relation to $\mathrm{K}$ at $\mathbf{x}$. We apply the same procedure to such diagrams at the second deciding crossing, and so on. The procedure ends with the last deciding crossing, thus yielding diagrams of unlinked colored links with $p+1$ crossings, where the function is given by (4), which depends only on the number of components and the number of colors. For an example see Sect. 1.2.

It remains to prove that:

(1) the procedure is independent of the order of the deciding points;

(2) the procedure is independent of the order of components and of the choice of base-points;

(3) the polynomial $F$ is invariant under Reidemeister moves.

Following the proof done in Lickorish and Millet (1987) for classical links, we observe that the proofs of statements (1), (2), and (3) can be done in an analogous way also in presence of colors. Of course, every time a skein relation is used, we have to pay attention to the colors of all links involved. We give here the proof of statement (1) as an example. The proofs of the other statements are similar.

The proof of statement (1) consists in a verification that the value of the invariant does not change if we interchange any two deciding crossings in the procedure of calculation. So, let $\mathrm{K}$ be the diagram of a colored link, and let $\mathrm{x}$ and $\mathrm{y}$ the first two deciding crossings that will be interchanged.

Denote by $\epsilon_{\mathrm{X}}$ the sign at the crossing $\mathrm{X}$, by $\sigma_{\mathrm{X}} \mathrm{K}$ the colored link diagram obtained from $K$ by changing the sign at the crossing $x$, by $\tilde{\sigma}_{x} K$ the diagram obtained from $K$ by changing the sign at $\mathrm{x}$ and coloring by a sole color all the components colored by the two colors of the two components crossing at $\mathrm{X}$; then denote by $\rho_{\mathrm{X}} \mathrm{K}$ the diagram obtained from $\mathrm{K}$ by removing the crossing $\mathrm{x}$ and coloring by a sole color all the components colored by the two colors of the components crossing at $\mathrm{x}$. Then do the same for the crossing $y$.

If $y$ follows $x$, then, by skein relations Va,b,

$$
F(\mathrm{~K})=w^{2 \epsilon_{\mathrm{x}}}\left[F\left(\sigma_{\mathrm{x}} \mathrm{K}\right)+\left(t^{\epsilon_{\mathrm{x}}}-1\right) F\left(\tilde{\sigma}_{\mathrm{x}} \mathrm{K}\right)\right]+w^{\epsilon_{\mathrm{x}}}\left(t^{\epsilon_{\mathrm{x}}}-1\right) F\left(\rho_{\mathrm{x}} \mathrm{K}\right),
$$


and

$$
\begin{aligned}
F(K)= & w^{2 \epsilon_{\mathrm{x}}}\left\{w^{2 \epsilon_{\mathrm{y}}}\left[F\left(\sigma_{\mathrm{y}} \sigma_{\mathrm{x}} \mathrm{K}\right)+\left(t^{\epsilon_{\mathrm{y}}}-1\right) F\left(\tilde{\sigma}_{\mathrm{y}} \sigma_{\mathrm{x}} \mathrm{K}\right)\right]+w^{\epsilon_{\mathrm{y}}}\left(t^{\epsilon_{\mathrm{y}}}-1\right) F\left(\rho_{\mathrm{y}} \sigma_{\mathrm{x}} \mathrm{K}\right)\right\} \\
& +\left(t^{\epsilon_{\mathrm{x}}}-1\right) w^{2 \epsilon_{\mathrm{x}}}\left\{w^{2 \epsilon_{\mathrm{y}}}\left[F\left(\sigma_{\mathrm{y}} \tilde{\sigma}_{\mathrm{x}} \mathrm{K}\right)+\left(t^{\epsilon_{\mathrm{y}}}-1\right) F\left(\tilde{\sigma}_{\mathrm{y}} \tilde{\sigma}_{\mathrm{x}} \mathrm{K}\right)\right]\right. \\
& \left.+w^{\epsilon_{\mathrm{y}}}\left(t^{\epsilon_{\mathrm{y}}}-1\right) F\left(\rho_{\mathrm{y}} \tilde{\sigma}_{\mathrm{x}} \mathrm{K}\right)\right\}+\left(t^{\epsilon_{\mathrm{x}}}-1\right) w^{\epsilon_{\mathrm{x}}}\left\{w ^ { 2 \epsilon _ { \mathrm { y } } } \left[F\left(\sigma_{\mathrm{y}} \rho_{\mathrm{x}} \mathrm{K}\right)\right.\right. \\
& \left.\left.\left.+t^{\epsilon_{\mathrm{y}}}-1\right) F\left(\tilde{\sigma}_{\mathrm{y}} \rho_{\mathrm{x}} \mathrm{K}\right)\right]+w^{\epsilon_{\mathrm{y}}}\left(t^{\epsilon_{\mathrm{y}}}-1\right) F\left(\rho_{\mathrm{y}} \rho_{\mathrm{x}} \mathrm{K}\right)\right\} .
\end{aligned}
$$

If $\mathrm{x}$ follows $\mathrm{y}$, then $F(\mathrm{~K})$ is obtained from the above expression by interchanging $\mathrm{x}$ with $y$. Observe that this expression contains terms of type $\tau_{\mathrm{y}} \tau_{\mathrm{x}} \mathrm{K}$ or $\alpha\left(\tau_{\mathrm{x}} \tau_{\mathrm{y}}^{\prime} \mathrm{K}+\tau_{\mathrm{x}}^{\prime} \tau_{\mathrm{y}} \mathrm{K}\right)$, where $\left(\tau, \tau^{\prime}\right) \in\{\sigma, \tilde{\sigma}, \rho\}$ and $\alpha$ is a coefficient. Such terms are invariant under the interchange of $\mathrm{x}$ with $\mathrm{y}$, because the operation $\tau_{\mathrm{x}}$ commutes with $\tau_{\mathrm{y}}$ as well as with $\tau_{\mathrm{y}}^{\prime}$ (since the procedures of coloring by a sole color the components of the two colors of the strands crossings respectively at $\mathrm{x}$ and at $\mathrm{y}$ produce equivalent colorations of the link if they are interchanged. Therefore, $F(\mathrm{~K})$ is independent of the order of $(\mathrm{x}, \mathrm{y})$.

\subsection{Properties of the Polynomial $F$}

Here we list some properties of the polynomial $F$, which can be easily verified.

(i) $F$ is multiplicative with respect to the connected sum of colored links.

(ii) The value of $F$ does not change if the orientations of all curves of the link are reversed.

(iii) Let $\mathrm{K}$ be a link diagram whose components are all tied together, and $\mathrm{K}^{ \pm}$be the link diagram obtained from $\mathrm{K}$ by changing the signs of all crossings. Then $F\left(\mathrm{~K}^{ \pm}\right)$ is obtained from $F(\mathrm{~K})$ by the following changes: $w \rightarrow 1 / w$ and $t \rightarrow 1 / t$.

(iv) Let $\mathrm{K}$ be a knot or a link, whose components have all the same color. Then $F(\mathrm{~K})$ is defined by relations I and IV, and is a HOMFLY polynomial with

$$
\ell=i \frac{1}{w \sqrt{t}} \text { and } m=i\left(\frac{1}{\sqrt{t}}-\sqrt{t}\right)
$$

In particular, for $w=t^{1 / 2}$ it becomes the Jones polynomial.

Statement (i) is deduced from defining relation I of $F$, by the same arguments that prove the multiplicativity of the invariants obtained by skein relations (see Lickorish and Millet 1987). Statement (ii) is evident, since the value of $F$ on the unlinked circles is independent of their orientation, and the skein relations are invariant under the inversion of the orientations of the strands. Statement (iii) follows from the fact that, if $w$ and $t$ are replaced by $1 / w$ and $1 / t$ respectively, then the skein relations $\mathrm{Va}$ and $\mathrm{Vb}$ are interchanged, whereas the term $y /(x w)$ stays unchanged.

To prove statement (iv), we compare skein relation IV, multiplied by $t^{1 / 2}$, with Eq. (1); this provides the expressions of $\ell$ and $m$ in terms of $x, y$, and $t$. Furthermore, since for the Jones polynomial $V(\mathrm{~K})$

$$
\ell=i t^{-1}, \quad \text { and } \quad m=i\left(t^{-1 / 2}-t^{1 / 2}\right),
$$


we observe that the expressions of $m$ are the same for $F(\mathrm{~K})$ and $V(\mathrm{~K})$, whereas the expressions of $\ell$ coincide under the equality $w=t^{1 / 2}$.

\subsection{An Example}

Let us apply the construction described in Theorem 1 to calculate the polynomial $F(\mathrm{~K})$ for the oriented colored link K shown in Fig. 3.

In Fig. 4, we show $\mathrm{K}$ with the deciding crossings and the associated diagrams $\mathrm{K}^{\prime}$ and $\tilde{\mathrm{K}}^{\prime}$.

The first deciding crossing is positive, the second one is negative. Applying relation Va to crossing I, we obtain (see Fig. 5):

$$
F(\mathrm{~K})=w^{2}\left[F\left(\mathrm{~K}_{1}\right)+(t-1) F\left(\mathrm{~K}_{2}\right)\right]+w(t-1) F\left(\mathrm{~K}_{3}\right) .
$$

Now we calculate $F\left(\mathrm{~K}_{1}\right)$ by applying relation $\mathrm{Vb}$ to crossing II of $\mathrm{K}_{1}$ :

$$
F\left(\mathrm{~K}_{1}\right)=w^{-2}\left[F\left(\mathrm{~K}_{4}\right)+\left(t^{-1}-1\right) F\left(\mathrm{~K}_{5}\right)\right]+w^{-1}\left(t^{-1}-1\right) F\left(\mathrm{~K}_{6}\right)
$$

then we calculate $F\left(\mathrm{~K}_{2}\right)$ by applying relation IV to crossing II of $\mathrm{K}_{2}$ :

$$
F\left(\mathrm{~K}_{2}\right)=t^{-1} w^{-2} F\left(K_{5}\right)+w^{-1}\left(t^{-1}-1\right) F\left(\mathrm{~K}_{6}\right) ;
$$

and calculate $F\left(\mathrm{~K}_{3}\right)$ by applying relation IV to crossing II of $\mathrm{K}_{3}$ :

$$
F\left(\mathrm{~K}_{3}\right)=t^{-1} w^{-2} F\left(\mathrm{~K}_{7}\right)+w^{-1}\left(t^{-1}-1\right) F\left(\mathrm{~K}_{8}\right) .
$$

Fig. 3 The components of $\mathrm{K}$ are numbered, and a base point is chosen on each of them

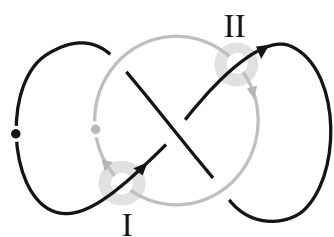

K

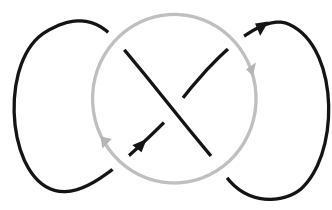

$K^{\prime}$
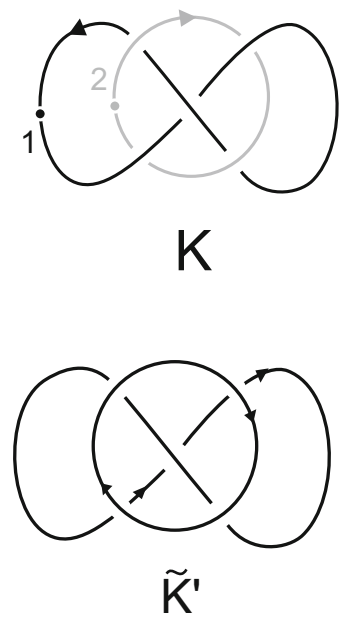

Fig. 4 The deciding crossings are marked as I and II 




Fig. 5 Calculation of $F(\mathrm{~K})$

Then we observe that:

$\mathrm{K}_{4}=\mathrm{K}^{\prime}$, and therefore, $F\left(\mathrm{~K}_{4}\right)=1 /(w x)$;

$\mathrm{K}_{5}=\tilde{\mathrm{K}}^{\prime}$, and therefore, $F\left(\mathrm{~K}_{5}\right)=y /(w x), y$ being given by (2);

$\mathrm{K}_{6}$ and $\mathrm{K}_{7}$ are both c-isotopic to $\mathrm{O}$, the unknotted circle; therefore, $F\left(\mathrm{~K}_{6}\right)=$ $F\left(\mathrm{~K}_{7}\right)=1$;

$\mathrm{K}_{8}$ and $\mathrm{K}_{2}$ are c-isotopic; indeed, they are different projections of two oriented circles of the same color linked by two negative crossings.

Therefore, by Eq. (7):

$$
F\left(\mathrm{~K}_{2}\right)=F\left(\mathrm{~K}_{8}\right)=\frac{w^{2} t^{2}-w^{2} t+w^{2}-1}{w^{3} t(1-t)} .
$$

Substituting the values of $F\left(K_{7}\right)$ and $F\left(K_{8}\right)$ in Eq. (8), we get

$$
F\left(\mathrm{~K}_{3}\right)=\frac{w^{2} t^{2}+w^{2}-1}{w^{4} t^{2}}
$$

Since, by Eq. (6), we have

$$
F\left(\mathrm{~K}_{1}\right)=\frac{w^{2} x+t-x}{w^{3} x t},
$$


we get, by Eq. (5):

$$
F(\mathrm{~K})=\frac{w^{4}\left(x t^{2}-x t^{3}\right)+w^{2}\left(t^{2}+x t-x-x t^{2}+x t^{3}\right)+x-x t}{w^{3} x t^{2}} .
$$

Observe also that $\mathrm{K}_{3}$ is c-isotopic to the oriented trefoil with three negative crossings, and that $\mathrm{K}_{1}$ is c-isotopic to two oriented circles of different colors linked by two negative crossings.

\section{References}

Aicardi, F., Juyumaya, J.: Markov trace on the algebra of braids and ties, to appear in Moscow Mathematical Journal. See also arXiv:1408.5672 (2014)

Aicardi, F., Juyumaya, J.: Tied links, to appear in JKTR, Slavik Jablan memorial volume. See also arXiv:1503.00527 (2015)

Alexander, J.W.: Topological invariants of knots and links. Trans. Am. Math. Soc. 30, 275-306 (1928)

Cimasoni, D.: A geometric construction of the Conway potential function. Comment. Math. Helv. 79, 124-146 (2004)

Cimasoni, D., Florens, V.: Generalized Seifert surfaces and signatures of colored links. Trans. Am. Math. Soc. 360(3), 1223-1264 (2008)

Freyd, P., Yetter, D., Hoste, J., Lickorish, W.B.R., Millet, K.C., Ocneanu, A.: A new polynomial invariant of knots and links. Bull. A.M.S. 12, 239-246 (1985)

Jones, V.F.R.: Hecke algebra representations of braid groups and link polynomials. Ann. Math. 126, 335-388 (1987)

Lickorish, W.B.R., Millet, K.C.: A polynomial invariant of oriented links. Topology 26(1), 107-141 (1987) Murakami, J.: On local relations to determine the multi-variable Alexander polynomial of colored links. In: Kawauchi, A (ed.) Knots, vol. 90, pp. 455-464 (Osaka, 1990). Walter de Gruyter, Berlin (1992) 